\title{
Characterization of the novel tumor-suppressor gene CCDC67 in papillary thyroid carcinoma
}

\author{
De-Tao Yin ${ }^{1,2, *}$, Jianhui $X \mathbf{u}^{1,2, *}$, Mengyuan Lei, ${ }^{1,2}$, Hongqiang Li $^{1,2}$, Yongfei Wang ${ }^{1,2}$, \\ Zhen Liu ${ }^{1,2}$, Yubing Zhou ${ }^{3}$, Mingzhao Xing ${ }^{4}$ \\ ${ }^{1}$ Department of Thyroid Surgery, The First Affiliated Hospital of Zhengzhou University, Zhengzhou, 450052, P. R. China \\ ${ }^{2}$ Key Discipline Laboratory of Clinical Medicine Henan, Zhengzhou 450052, P. R. China \\ ${ }^{3}$ Department of Pharmacy, The First Affiliated Hospital of Zhengzhou University, Zhengzhou, 450052, P. R. China \\ ${ }^{4}$ Division of Endocrinology and Metabolism, the Johns Hopkins University School of Medicine, Baltimore, MD 21287, USA \\ *These authors contributed equally to this work \\ Correspondence to: De-tao Yin, e-mail: detaoyin@zzu.edu.cn \\ Mingzhao Xing, e-mail: mxing1@jhmi.edu \\ Keywords: thyroid cancer, CCDC67, tumor suppressor, prognostic marker, molecular pathogenesis
}

Received: September 01, 2015

Accepted: November 25, 2015

Published: December 22, 2015

\section{ABSTRACT}

Background: Some studies showed an association of coiled-coil domain-containing $(C C D C)$ genes with cancers. Our previous limited data specifically suggested a possible pathogenic role of CCDC67 in papillary thyroid cancer (PTC), but this has not been firmly established. The present study was to further investigate and establish this role of CCDC67 in PTC.

Results: The expression of CCDC67, both at mRNA and protein levels, was sharply down-regulated in PTC compared with normal thyroid tissues. Lower CCDC67 expression was significantly associated with aggressive tumor behaviors, such as advanced tumor stages and lymph node metastasis, as well as BRAF mutation. Introduced expression of CCDC67 in TPC-1 cells robustly inhibited cell proliferation, colony formation and migration, induced G1 phase cell cycle arrest, and increased cell apoptosis.

Methods: Primary PTC tumors and matched normal thyroid tissues were obtained from 200 unselected patients at the initial surgery for detection of CCDC67 mRNA and protein by RT-PCR and Western blotting analyses, respectively. Genomic DNA sequencing was performed to detect BRAF mutation in PTC tumors. Clinicopathological data were retrospectively reviewed for correlation analyses. PTC cell line TPC-1 with stable transfection of CCDC67 was used to investigate the functions of CCDC67.

Conclusions: This large study demonstrates down-regulation of CCDC67 in PTC, an inverse relationship between CCDC67 expression and PTC aggressiveness and BRAF mutation, and a robust inhibitory effect of CCDC67 on PTC cellular activities. These results are consistent with $C C D C 67$ being a novel and impaired tumor suppressor gene in PTC, providing important prognostic and therapeutic implications for this cancer.

\section{INTRODUCTION}

Thyroid carcinoma is a common endocrine malignancy that has seen a rapid increase in incidence globally in recent decades and is, in fact, currently the most rapidly increasing cancer among women and the second most among men $[1,2]$. Papillary thyroid carcinoma (PTC) is the most common type of thyroid malignancy, accounting for over
$90 \%$ of all thyroid malignancies [3]. Thyroid carcinoma is predominantly driven by genetic and epigenetic alterations, including activation of oncogenes and inactivation of tumor suppressor genes $[4,5]$. Much still remains unknown about the molecular mechanisms of PTC even though many genetic and epigenetic alterations have been known in thyroid carcinoma, such as aberrant hypermethylation of $\mathrm{CpG}$ islands in promoter regions of tumor suppressor genes (TSGs) [6, 7]. 
The coiled-coil domain-containing $(C C D C)$ proteins exhibit diverse functions related to their highly versatile folding motif [8]. The coiled-coil motif is found in many proteins, such as skeletal and motor proteins, and is involved in molecular recognition systems and protein refolding [9]. Previous studies have shown genetic or epigenetic alterations in several $C C D C$ genes in human cancers, including CCDC19 (NESG1) in nasopharyngeal carcinoma [10], CCDC62 in prostate cancer [11], $C C D C 116$ in pancreatic cancer [12], CCDC6 in lung cancer [13], CCDC8 in renal carcinoma [14], CCDC98 in breast cancer [15], and $C C D C 134$ in gastric cancer [16]. CCDC67 is located in chromosome 11q21 and encodes a 604 amino acid protein containing a coiled-coil domain, including 3B79 (GenBank accession no.CG464599) clone sites with NotI/EcoRV segments covering the 5 'upstream area, which are associated with cell signal transduction in tumor formation $[17,18]$. Park [18] found down-regulation and aberrant methylation of CCDC67 in gastric cancer.

The role of CCDC67 in thyroid carcinoma has not been well investigated except for a recent preliminary study on a limited cohort of patients in which we observed a downregulation of CCDC67 mRNA in PTC and an association of this down-regulation with some aggressive features of PTC [19]. Although this finding seems to suggest that CCDC67 is a novel tumor suppressor gene in PTC, no firm conclusion could be established due to the limited scope of the study. Functional data is also lacking regarding a tumor suppressor role of this gene in PTC. In the present study on a large cohort of patients, we explored further to establish CCDC67 as a novel tumor suppressor gene in PTC.

\section{RESULTS}

\section{Down-regulation of $C$ CDC67 expression in PTC and tumor aggressiveness}

To firmly establish the clinicopathological role of CCDC67 in PTC, here we dramatically expanded the cohort of patients to 200 cases from our previous small cohort [19] and additionally examined protein expression of CCDC67 (previously only mRNA was examined). RT-PCR and Western blot were used to detect the expression of CCDC67 in 200 PTC samples and their matched adjacent normal thyroid tissues. Representative results are shown in Figure 1. As shown in Figure 1A, some cases showed expression of CCDC67 mRNA and some did not. All the 200 matched normal tissues showed robust expression of CCDC67 mRNA while only $47.0 \%$ (94/200) cases of PTC tumors expressed CCDC67 mRNA expression. Similar pattern was seen with the expression of $C C D C 67$ protein (Figure 1B). All the 200 samples of normal tissues expressed CCDC67 protein while only $41.5 \%(83 / 200)$ cases of their matched PTC tumors expressed CCDC67 protein. There was a strong correlation between the
mRNA and protein expression of the CCDC67 gene $\left(\chi^{2}=33.038, P<0.01\right)$ (Table 1). Interestingly, lack of CCDC67 mRNA expression was associated with more aggressive tumor stages and lymph node metastasis (Table 2). Specifically, a dramatically higher number of cases of PTC without CCDC67 mRNA expression displayed high TNM stages III/IV, pathological grade II, and lymph node metastasis, with $\chi^{2}=8.236,4.236$, and 4.322, respectively, and $P<0.05$ in all cases. A similar significant association was seen between the lack of CCDC67 protein expression and high TNM stages III/ IV, pathological grade II, and lymph node metastasis, with $\chi^{2}=3.860,5.548$, and 7.275, respectively, and $P<0.05$ in all cases (Table 2).

\section{Association between the down-regulation of CCDC67 expression and the presence of BRAF T1799A mutation}

One hundred sixty-eight of the 200 PTC tissues were available for genomic DNA isolation and analysis for $B R A F$ T1799A mutation. The BRAF mutation was found only in tumor samples but not in their normal counterparts. Figure 2 shows a representative electropherogram of the $B R A F$ 1799A mutation. We found that $42.3 \%(71 / 168)$ cases of PTC expressed CCDC67 mRNA and 39.3\% (66/168) cases of PTC harbored the BRAF mutation. There was a strong association between the down-regulation of CCDC67 expression and the presence of the BRAF mutation $\left(\chi^{2}=17.000, P<0.01\right.$, Table 3$)$.

\section{Overexpression of $C$ CDC67 suppressed TPC-1 cell proliferation and colony formation}

The above data strongly support CCDC67 being a tumor suppressor gene. To further test this, we used TPC- 1 cells, which naturally lack expression of CCDC67 as we previously demonstrated [19] and as shown in Figure 3A, to examine the effect of introduced expression of CCDC67 on cellular activities. Stably transfected CCDC67-expressing TPC-1 cells were established using a GFP-tagged CCDC67 expression vector (Figure 3A). The effect of $C C D C 67$ on cell proliferation was examined using the CCK-8 assay and a significant inhibition of cell proliferation by induced CCDC67 expression compared with the control (transfection with empty vector (NC) was observed (Figure 3B). Cell colony formation was also significantly inhibited by CCDC67 overexpression (Figure 3C).

\section{CCDC67 inhibited the migration and invasion of TPC-1 cells}

We next tested whether CCDC67 could inhibit the migratory and invasive abilities of TPC-1 cells by performing the wound healing (cell migration) assay 
and the migration and transwell invasion assays. The wound healing ability (migratory ability) of cells was significantly decreased in TPC-1 cells with introduced overexpression of CCDC67 compared with the control cells transfected only with the empty vector (Figure 4A and Figure 4B). The migration and transwell invasion assays showed that CCDC67 overexpression also significantly suppressed the migration (Figure 5A) and invasion (Figure 5B) of TPC-1 cells as evidenced by a significant decrease in the number of migrating and invading cells, respectively (both $P<0.01$ ).

\section{Overexpression of $C C D C 67$ induced $\mathrm{G} 1$ cell cycle arrest and promoted apoptosis of TPC-1 cells}

We also examined the effect of CCDC67 on cell cycle distribution using flow cytometry. As shown in Figure 6A, compared with the control, introduced expression of CCDC67 in TPC-1 cells promoted a significant shift of the cell cycle to the G0/G1 phase from the $\mathrm{S}$ phase. The apoptosis rate of cells was also significantly increased by introduced expression of CCDC67 (Figure 6B).

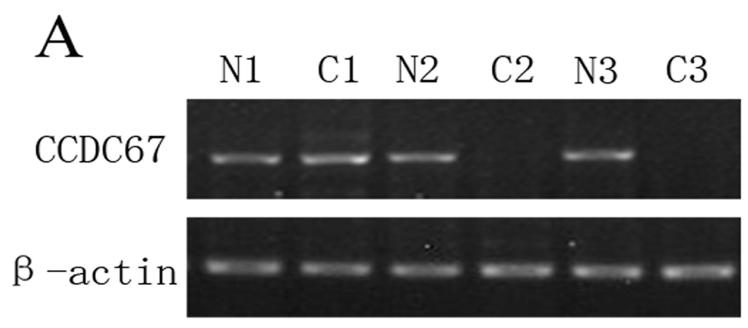

\section{DISCUSSION}

The coiled-coil motif of proteins is important for molecular recognition in the regulation of cellular functions and activities, through which these proteins may play an important role in human tumorigenesis [20-24]. One of such proteins, CCDC67, was previously indicated to play a possible suppressor role in tumor metastasis [25]. The promoter of the CCDC67 gene was found to be hypermethylated in gastric cancer [18]. This is consistent with CCDC67 being a tumor suppressor gene since, in addition to genetic impairment, such as genetic deletion and loss-of-function mutations, epigenetic alteration in the form of DNA methylation of the promoter regions is also an important mechanism in the inactivation of tumor suppressor genes [26-28]. The tumor suppressor role of the CCDC67 gene, however, has not been firmly established. Its role in thyroid tumorigenesis is completely unknown. In the present study we investigated the potential tumor suppressor function of CCDC67 specifically in thyroid cancer.

In the present analysis of a large cohort of 200 patients with PTC, we demonstrated a down-regulation

Figure 1: Expression status of CCDC67 in PTC tumors (C) and matched adjacent normal thyroid tissues (N). (A) Shown are examples, representing 200 patients with papillary thyroid cancer, of RT-PCR analysis of CCDC67 mRNA expression in PTC and adjacent tissues. (B) Western blot analysis of CCDC67 protein in PTC and adjacent normal tissues. N, matched normal thyroid tissue; $\mathrm{C}$, cancer tissue.
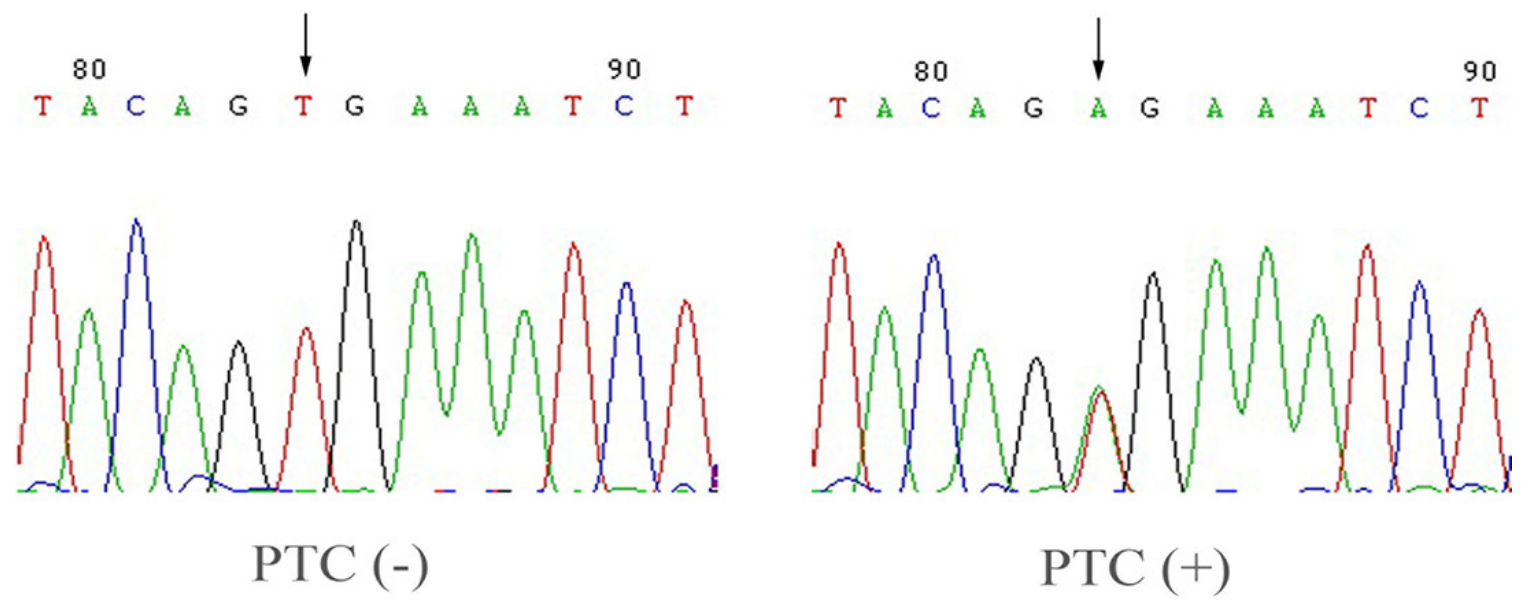

Figure 2: Detection of BRAF T1799A in PTC. Shown is the representative electropherogram of the hot spot in exon 15 of the $B R A F$ gene carrying the BRAF T1799A mutation. A case of PTC with the wild-type BRAF (PTC-) and a case with the BRAF T1799A mutation $(\mathrm{PTC}+)$ are shown. The arrow indicates the nucleotide where the mutation occurs. 
Table 1: Relationship between mRNA and protein expression of $C C D C 67$

\begin{tabular}{|c|c|c|c|}
\hline \multirow[t]{2}{*}{$C C D C 67$ protein } & \multicolumn{2}{|c|}{ CCDC67 mRNA } & \multirow[t]{2}{*}{ Total } \\
\hline & + & - & \\
\hline+ & 59 & 35 & 94 \\
\hline- & 24 & 82 & 106 \\
\hline Total & 83 & 117 & 200 \\
\hline
\end{tabular}

$\chi^{2}=33.038, P<0.01$.

Table 2: The relationship between $C C D C 67$ expression and clinicopathological features in PTC

\begin{tabular}{|c|c|c|c|c|c|c|}
\hline \multirow[t]{2}{*}{ Clinical date } & \multicolumn{2}{|c|}{ Expression of mRNA } & \multirow{2}{*}{$\boldsymbol{P}$} & \multicolumn{2}{|c|}{ Expression of protein } & \multirow{2}{*}{$\boldsymbol{P}$} \\
\hline & Positive & Negative & & Positive & Negative & \\
\hline \multicolumn{7}{|l|}{ Sex } \\
\hline male & 37 & 47 & 0.477 & 34 & 50 & 0.803 \\
\hline female & 57 & 59 & & 49 & 67 & \\
\hline \multicolumn{7}{|l|}{ Age } \\
\hline$<45$ & 40 & 56 & 0.147 & 36 & 60 & 0.270 \\
\hline$\geq 45$ & 54 & 50 & & 47 & 57 & \\
\hline \multicolumn{7}{|l|}{ Tumor size } \\
\hline$<2 \mathrm{~cm}$ & 57 & 51 & 0.076 & 49 & 59 & 0.229 \\
\hline$\geq 2 \mathrm{~cm}$ & 37 & 55 & & 34 & 58 & \\
\hline \multicolumn{7}{|l|}{ TNM stage } \\
\hline $\mathrm{I} \sim \mathrm{II}$ & 59 & 45 & 0.004 & 50 & 54 & 0.049 \\
\hline III IV & 35 & 61 & & 33 & 63 & \\
\hline \multicolumn{7}{|l|}{ Pathological grade } \\
\hline I & 58 & 50 & 0.040 & 53 & 55 & 0.019 \\
\hline II & 36 & 56 & & 30 & 62 & \\
\hline \multicolumn{7}{|c|}{ Lymph node metastasis } \\
\hline $\mathrm{NO}$ & 59 & 51 & 0.038 & 55 & 55 & 0.007 \\
\hline YES & 35 & 55 & & 28 & 62 & \\
\hline Total & 94 & 106 & & 83 & 117 & \\
\hline
\end{tabular}

of CCDC67 expression in PTC tumors compared with normal thyroid tissues. We also demonstrated an association between tumor aggressiveness and decreased expression of $C C D C 67$. These results were consistent with CCDC67 being a tumor suppressor gene in PTC, which prompted us to take the next step to functionally study the CCDC67 gene in PTC cells. Indeed, using a variety of cellular and molecular approaches, we demonstrated a remarkable suppressing effect of introduced expression of CCDC67 on several cellular activities, such as cell proliferation, colony formation, migration, invasion, and tumor spheroid formation. These results are all well consistent with $C C D C 67$ being a tumor suppressor gene in thyroid cancer. Our finding of the strong pro-apoptotic function of $C C D C 67$ is in line with the recent report that $\mathrm{CCDC}$ proteins play a role in the cellular apoptotic process [29]. The role of CCDC67 in thyroid cancer cellular activities observed in the present study is likely influenced by the microenvironments as studies in recent years have demonstrated that the latter play a critical role in thyroid cancer cell behavior [30-32].

The molecular pathogenesis of thyroid cancer involves genetic alterations in both oncogenes and tumor suppressor genes, as exemplified by $B R A F$ and $R A S$ for the former and PTEN [1] and RASAL1 [33] for the latter. Our finding in the present study of the association between down-regulation of CCDC67 and BRAF mutation in PTC suggests that the latter might play a role in the negative regulation of the former. Identification of CCDC67 as a novel tumor suppressor gene in thyroid cancer expands the genetic repertoire for thyroid cancer. The specific mechanisms involved in the tumor suppressor role of CCDC67 and whether there is genetic or epigenetic alteration in the $C C D C 67$ gene in thyroid cancer remains to be investigated. Nevertheless, the clinicopathological data and the cellular functional data in the present 


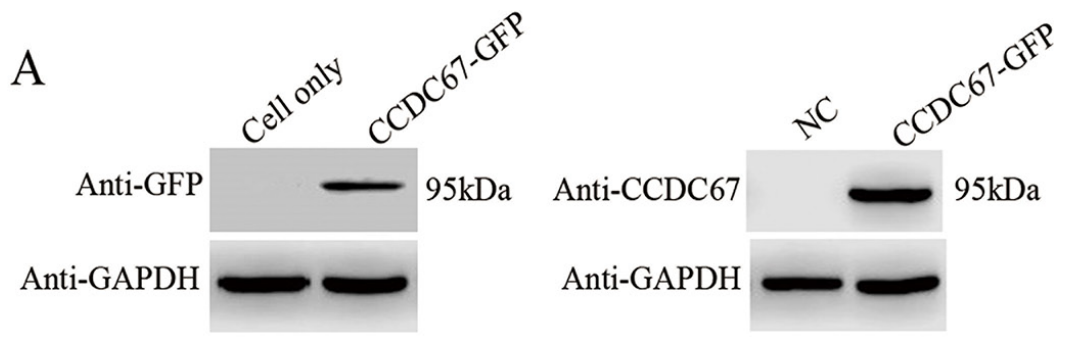

B

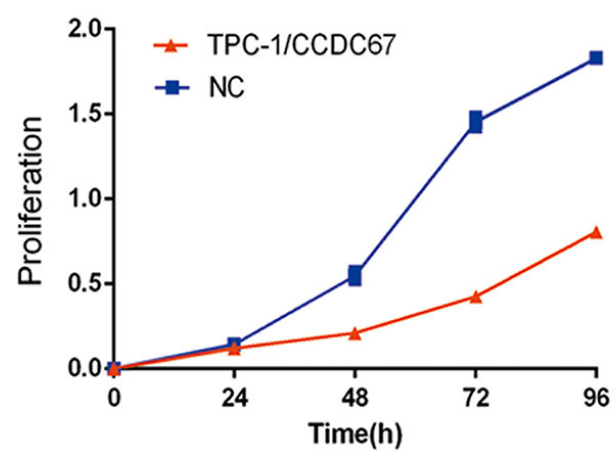

$\mathrm{C}$

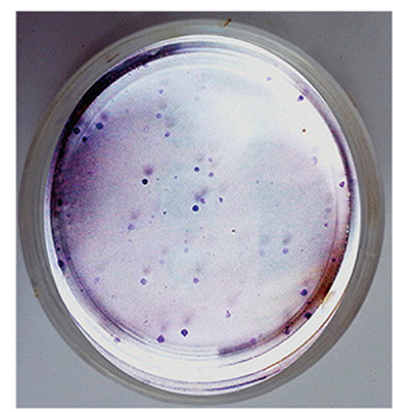

$\mathrm{NC}$

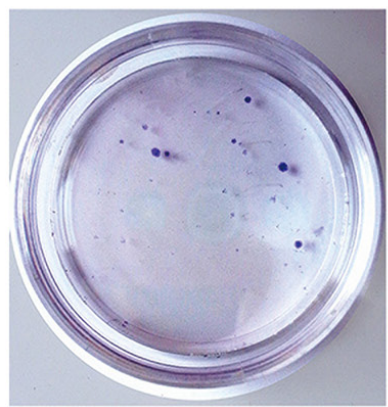

TPC-1/CCDC67

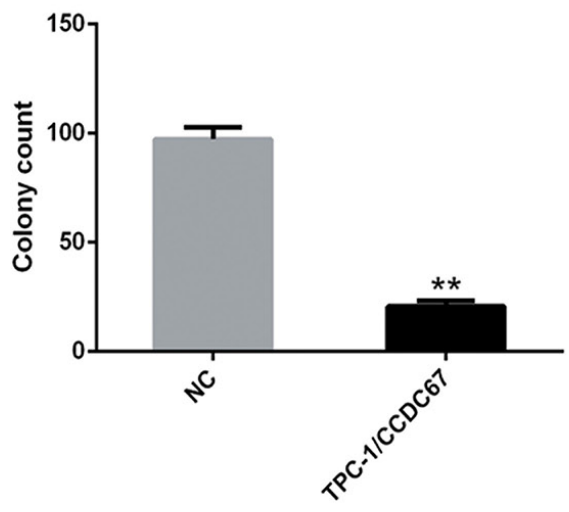

Figure 3: Introduced overexpression of CCDC67 suppressed TPC-1 cell proliferation and colony formation. (A) Introduced expression of CCDC67 in transfected PTC-1 cells. TPC-1 cells, which naturally lacked CCDC67 expression, were transfected with the CCDC67-GFP expressing vector or the empty vector as control (NC). Western blotting was performed using anti-GFP or anti-CCDC67 antibodies. GAPDH protein was tested as a control. (B) Cell proliferation assay. TPC-1 cells expressing CCDC67 or control cells (NC) were cultured in 96-well plates for the indicated time (1-4 days), and cell proliferation was measured using a CCK-8 kit. Proliferation was significantly decreased in TPC-1 cells expressing CCDC67 compared with NC. (C) Adhesion-dependent colonyformation assay in monolayer culture. TPC-1 cells expressing CCDC67 and NC cells were plated in six-well plates at 2000 cells/well. After 2 weeks of incubation, cells were stained with $0.1 \%$ crystal violet. Representative images are shown. In the bar graphs, the columns represent the mean colony number from three independent experiments and the little vertical bars on the top of the columns represent SD. ${ }^{* *} P<0.01$ in comparison with the control per Student's $t$ test. 
Table 3: Relationship between $C C D C 67$ mRNA expression and $B R A F$ mutation

\begin{tabular}{|c|c|c|c|}
\hline \multirow[t]{2}{*}{$B R A F$ mutation } & \multicolumn{2}{|c|}{ CCDC67 mRNA } & \multirow[t]{2}{*}{ Total } \\
\hline & + & - & \\
\hline+ & 15 & 51 & 66 \\
\hline- & 56 & 46 & 102 \\
\hline Total & 71 & 97 & 168 \\
\hline
\end{tabular}

$\chi^{2}=17.000, P<0.01$.
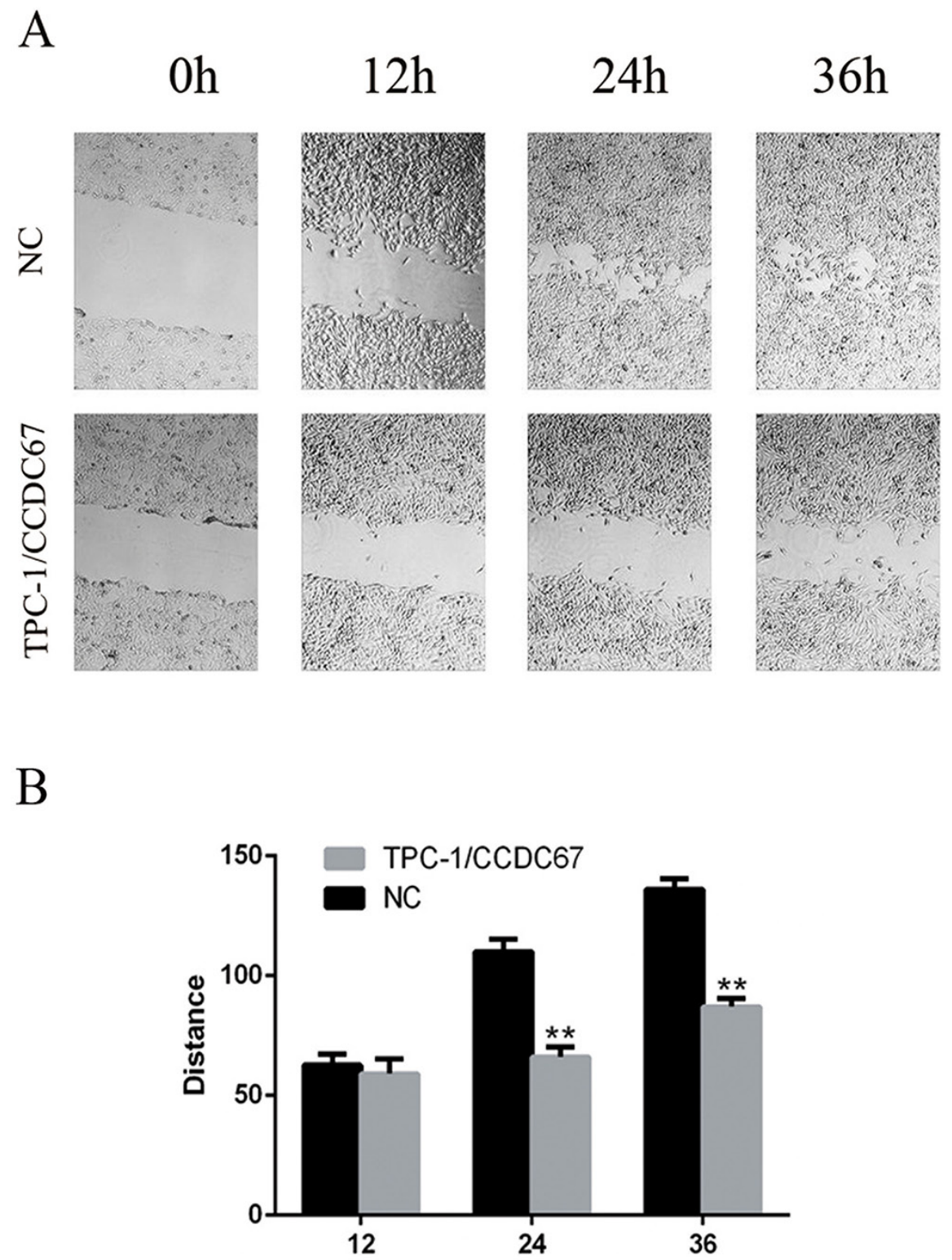

Figure 4: Wound healing assay of TPC-1 cell migration. (A) Representative images of wound healing (cell migration) as described in the Methods in TPC-1 cells with induced overexpression of CCDC67 (TPC-1/CCDC67) and control TPC-1 cells transfected with the empty vector (NC). Suppression of wound healing by CCDC67 is clearly visible. (B) Bar graph presentation of quantitative measurements of cell migration distance in PTC-1 cells over-expressing CCDC67 (TPC-1/CCDC67) and control cells (NC). Columns represent the mean of at least 3 independent experiments and the little vertical bars at the top of the columns represent $\mathrm{SD}$. ${ }^{* *} P<0.01$ in comparison with the control per Student's $t$ test. 
A

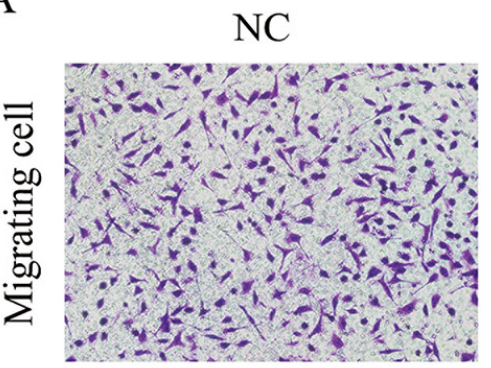

TPC-1/CCDC67
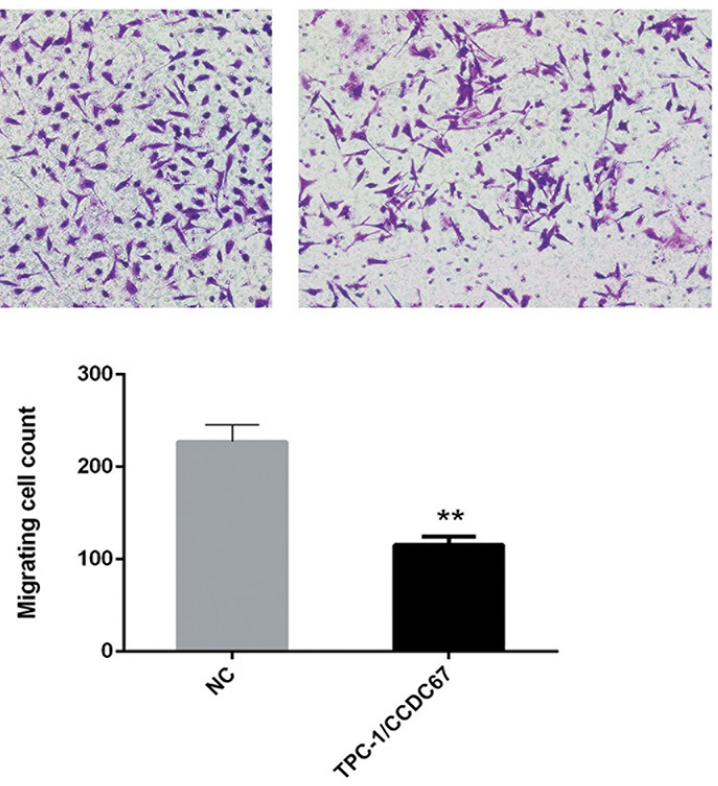

$\mathrm{B}$
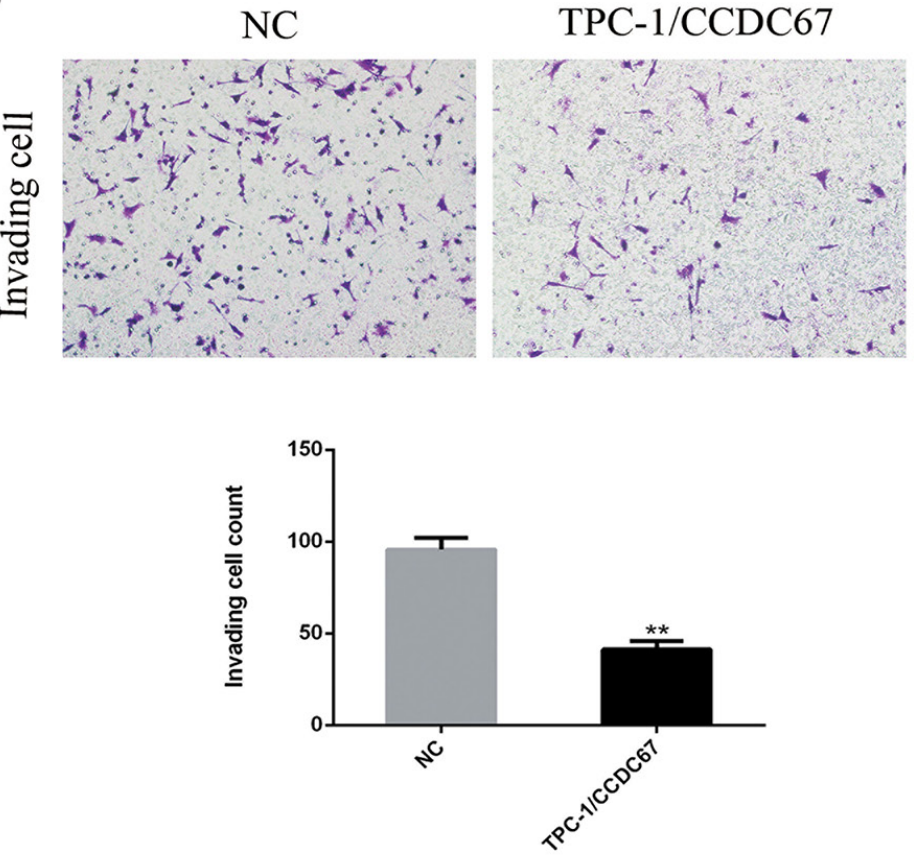

Figure 5: Migration and transwell invasion assays of TPC-1 cells. (A) Migration assay. Shown in the top panel are representative microphotograph images of migrating TPC-1 cells transfected with the empty vector (NC) or cells with stably introduced expression of CCDC67 (TPC-1/CCDC67). CCDC67 (TPC-1/CCDC67) cells had a reduced ability to cross the transwell membrane compared to NC cells as demonstrated by the weaker staining of the transwells in which they were seeded. Shown in the lower panel is the bar graph presentation of quantitative measurements of cell migration in control TPC-1 cells (NC) and TPC-1 cells over-expressing CCDC67 (TPC1/CCDC67). Columns represent the mean of the counts of migrating cell numbers from at least 3 independent experiments and the little vertical bars at the top of the columns represent SD. (B) Invasion assay. Shown in the top panel are representative microphotograph images of invading TPC-1 cells transfected with the empty vector (NC) or cells with stably introduced expression of CCDC67 (TPC-1/CCDC67). CCDC67 (TPC-1/CCDC67) cells had a reduced ability to cross the transwell membrane with Matrigel matrix compared to NC cells as demonstrated by the weaker staining of the transwells in which they were seeded. Shown in the lower panel is the bar graph presentation of quantitative measurements of cell invasion in control TPC-1 cells (NC) and TPC-1 cells over-expressing CCDC67 (TPC-1/CCDC67). Columns represent the mean of the counts of invading cell numbers from at least 3 independent experiments and the little vertical bars at the top of the columns represent SD. ${ }^{* *} P<0.01$ in comparison with the control per Student's $t$ test in both panels A and B. 
A

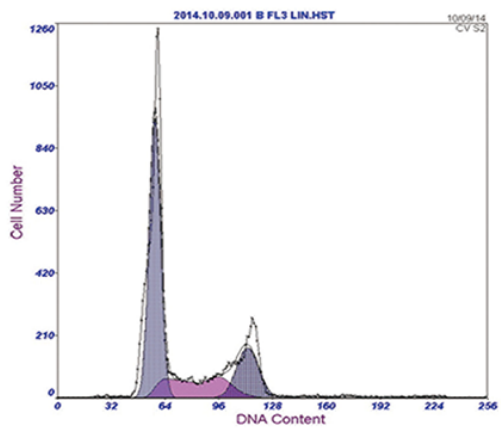

$\mathrm{NC}$

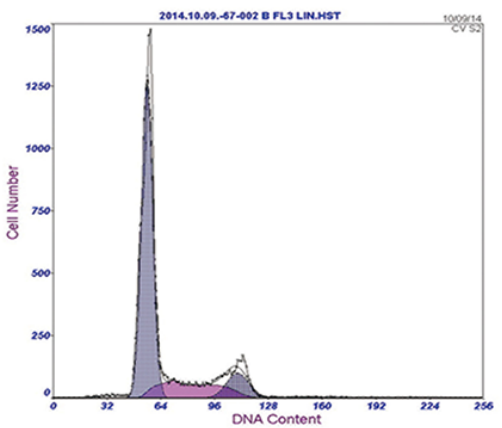

TPC-1/CCDC67

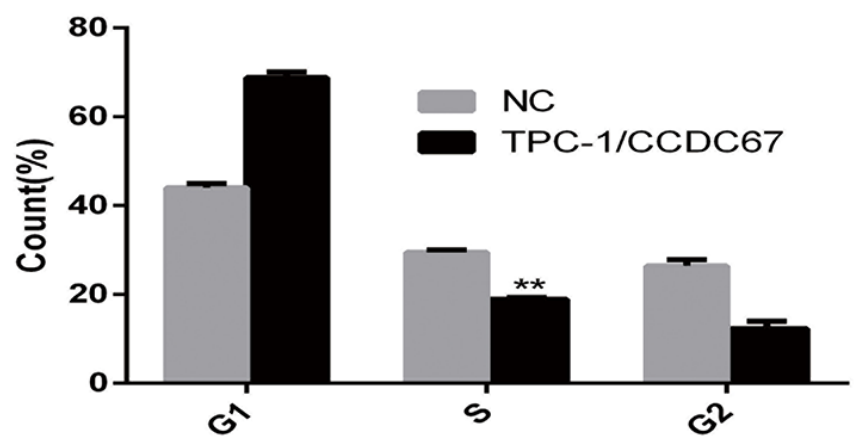

B
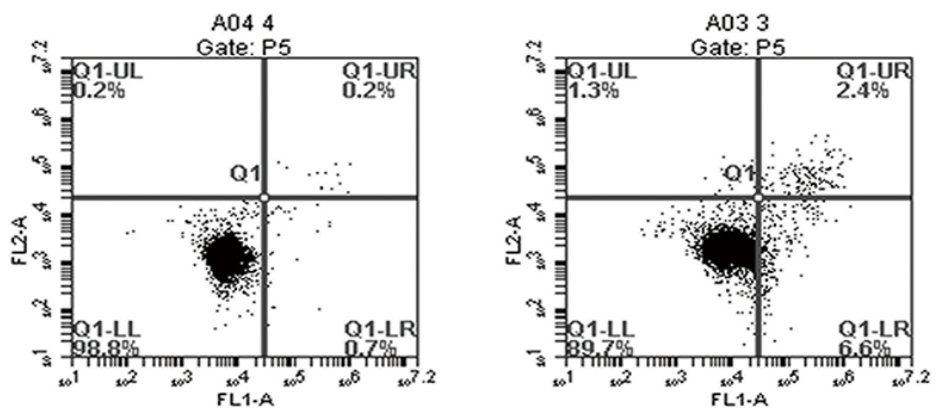

$\mathrm{NC}$

TPC-1/CCDC67

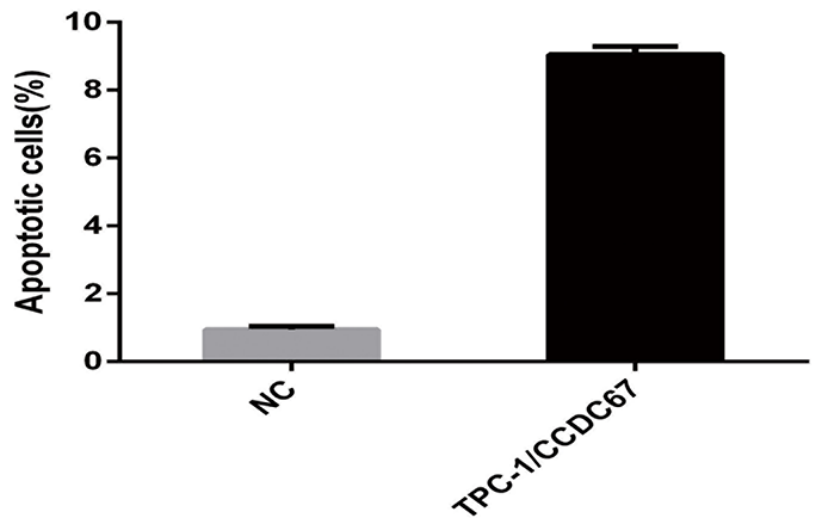

Figure 6: Effects of $\boldsymbol{C C D C 6 7}$ on cell cycle and apoptosis of TPC-1 cells. (A) Compared with the control cells (NC), TPC-1 cells with introduced expression of CCDC67 (TPC-1/CCDC67) displayed a significant increase in the G1 phase and decrease in the S phase in the cell cycle. The upper panel shows the distribution of cell cycle phases and the lower panel shows the quantitative measurements of the cell cycle phases. (B) Introduced expression of CCDC67 promoted cell apoptosis. The upper panel shows the extent of the apoptotic component of cells and the lower panels showed the quantitative measurements of the apoptotic cells. In the bar graphs, the columns represent mean of at least 3 independent experiments and the little vertical bars on the top of the columns represent $\mathrm{SD}$. ${ }^{* *} P<0.01$ in comparison with controls per Student's $t$-test. 
study are sufficient to establish that $C C D C 67$ is a novel tumor suppressor gene in thyroid cancer. Adding to our understanding of the molecular pathogenesis of thyroid cancer, these findings have important biological and clinical implications for this cancer.

\section{MATERIALS AND METHODS}

\section{Patients and tissue samples}

Two hundreds primary PTC samples and matched adjacent normal thyroid tissue samples were obtained at the time of initial surgery and snap-frozen immediately after tumor removal. The study was approved by the ethics committee of Zhengzhou University and human tissues were obtained with informed written consents from the patients. All tissue samples were reviewed by an endocrine pathologist to confirm the diagnosis. PTC samples estimated to contain more than $80 \%$ tumor cells were used.

\section{Cell lines and cell culture}

The TPC-1 cell line was kindly provided by Dr Ye Lei of Shanghai Rui Jin Hospital, Shanghai, China. This cell was cultured in RPMI1640 supplemented with 10\% fetal bovine serum and incubated at $37^{\circ} \mathrm{C}$ in a humidified atmosphere containing $5 \% \mathrm{CO}_{2}$.

\section{RT- PCR analysis}

Total RNA was extracted from PTC tissues and cell lines using the Trizol reagent (Invitrogen, USA). Total RNA $(1 \mu \mathrm{g})$ was reverse-transcribed to cDNA using PrimeScript ${ }^{\mathrm{TM}}$ II kit (Takara, Japan) according to the manufacturer' instructions. One $\mu 1$ of the resulting cDNA was used as the template for PCR in a $25-\mu 1$ reaction volume containing $12.5 \mu 1$ Premix Taq

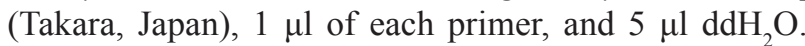
The $\beta$-actin mRNA level was used for normalization. PCR primer sequences are as follows-for $\beta$-actin: 5'-CTAAGTCATAGTCCGCCTAGAAGCA-3'(forward) and 5 '-TGGCACCCAGCACAATGAA-3'(reverse); for CCDC67: 5'-GCAGCTCTGAAATTCCTCGT-3' (forward) and 5'-TTGGTTGATCTTGCATCACTG-3' (reverse). The PCR cycles were performed as follows: one cycle at $95^{\circ} \mathrm{C}$ for $3 \mathrm{~min}, 40$ cycles at $95^{\circ} \mathrm{C}$ for $30 \mathrm{~s}$, $60^{\circ} \mathrm{C}$ for $30 \mathrm{~s}$ and $72^{\circ} \mathrm{C}$ for $30 \mathrm{~s}$, and one final cycle at $72^{\circ} \mathrm{C}$ for $10 \mathrm{~min}$, followed by cooling to $4^{\circ} \mathrm{C}$. Amplification products were separated on $3 \%$ agarose gels and photographed with a UV gel imaging system (Kodak, USA). Imagemaster DVS system was used to determine the relative mean gray values (A) of the target product and $\beta$-actin. Expression index (I) of the target product was calculated using the formula of $\mathrm{I}=\mathrm{A}$ product $/ \mathrm{A} \beta$-actin as previously described [34].

\section{Detection of $B R A F$ mutation in PTC}

The BRAF T1799A mutation was analyzed on genomic DNA isolated from 168 cases of primary PTC tissue samples that were available for DNA isolation. DNA was extracted from frozen tissue with the TaKaRa MiniBEST Universal Genomic DNA Extraction Kit (TaKaRa, Japan), according to the manufacturer's instructions.

$B R A F$ gene mutation was detected by direct genomic DNA sequencing analysis. To this end, a 212-bp fragment from exon 15 containing the site where T1799A mutation occurs was amplified by PCR. The primers used were as described previously [35]: TCATAATGCTTGCTCTGATAGGA (forward) and GGCCAAAAATTTAATCAGTGGA (reverse). The amplification PCR was performed with deoxynucleotides using a step-down protocol: $98^{\circ} \mathrm{C}$ for $10 \mathrm{~s}, 55^{\circ} \mathrm{C}$ for $15 \mathrm{~s}$, and $68^{\circ} \mathrm{C}$ for $1 \mathrm{~min}$ for 30 cycles. The reaction mixture contained about $80 \mathrm{ng}$ genomic DNA, $10 \mu \mathrm{l}$ $5 \times$ PrimeSTAR GXL Buffer, $4 \mu \mathrm{l}$ dNTP Mixture $(2.5 \mathrm{nM})$, $31 \mu \mathrm{lddH_{2 }} \mathrm{O}, 1 \mu \mathrm{l}$ of each primer (forward and reverse), and $1 \mu \mathrm{l}$ PrimeSTAR GXL DNA polymerase(TaKaRa, Japan) in a $50 \mu \mathrm{l}$ final volume. After confirmation of the efficiency and quality of the amplification PCR by running the PCR products on a $2.0 \%$ agarose gel, the PCR products were were purified by DNA Gel Extraction Kit (TaKaRa, Japan) and subjected to direct sequencing PCR using the above-described forward primer. The purified PCR products were sequenced by Lifetech Company in Shanghai, China.

\section{Western blot analysis}

For Western blotting, $30 \mathrm{mg}$ of fresh frozen tumor or the adjacent normal tissue or treated cells were lysed in RIPA lysis buffer (Cwbiotech, China) containing 1\% Protease Inhibitor Cocktail (Cwbiotech, China). An equal amount of protein of about $30 \mu \mathrm{g}$ was separated by sodium dodecyl sulfate polyacrylamide gel electrophoresis (SDS-PAGE) and transferred onto the poly vinylidene fluoride (PVDF) membrane. After blocking with 5\% skimmed milk, the PVDF membrane was incubated with anti-CCDC67 antibody (Abcam, USA). After washing three times with Tris-Buffered Saline and Tween 20, the membrane was incubated with horseradish peroxidase-linked secondary anti-mouse $\mathrm{IgG}$ antibody (Abcam, USA) at room temperature for $1 \mathrm{~h}$, followed by visualization using an ECL detection kit (Millipore, Billerica, MA). GAPDH protein, detected using an anti-GAPDH antibody purchased from Abcam, was used for loading control. Imagemaster DVS system was used to determine the relative mean gray values (A) of the target product and GAPDH. Expression index (I) of the target product was calculated using the formula of $\mathrm{I}=\mathrm{A}$ product/A GAPDH [34]. 


\section{Construction of lentivirus expression vector of CCDC67 and cell transfection}

To construct the lentivirus vector expressing $C C D C 67$, we amplified a cDNA fragment containing CCDC67 precursor from TPC-1 cells (forward: 5'-GAGGATCCCCGGGTACCGGTCGCCACCATGGAGA ACCAAGCCCATAATAC-3', reverse: 5'-TCACCATGGTG GCGACCGGTATGTGTCTATTTTGTTTTAGC-3'). The amplified fragment was cloned into a modified pGV208-GFP vector (Genechem, China). A day before lentivirus infection, TPC-1 cells in normal culture condition were seeded into six-well culture plates and grown overnight. Cells were then transfected with the pGV208-GFP-CCDC67 vector (defined as TPC-1/CCDC67) according to the multiplicity of infection $(\mathrm{MOI}=1)$. The empty vector pGV208-GFP was used for negative control (defined as NC). After an incubation for $96 \mathrm{~h}$, detection of the expression was performed under fluorescence.

\section{Cell proliferation assay and colony forming assay}

For the proliferation assay, TPC-1/CCDC67 and $\mathrm{NC}$ cells were seeded at $5 \times 10^{3}$ cells/well in 96-well plates and incubated at $37^{\circ} \mathrm{C}$ for 4 days. An aliquot of $10 \mu \mathrm{C}$ Cell Counting Kit-8 reagent (Dojindo, Japan) was added to the cells and, following a 3-h incubation, absorbance was measured at $450 \mathrm{~nm}$ using a spectrophotometer (Bio-Rad, USA).

For the colony forming assay, the two transfectant cells were plated at 100 cells/well in six-well plates, incubated for 2 weeks in RPMI-1640 containing puromycin $(1 \mu \mathrm{g} / \mathrm{ml})$, and stained with crystal violet. Colonies were photographed and counted after 3 weeks of incubation at $37^{\circ} \mathrm{C}$. All assays were performed in triplicate.

\section{Migration and invasion assay}

For migration assay, $200 \mu \mathrm{l}$ of the TPC-1/CCDC67 and NC cells in serum-free RPIM-1640 were seeded on upper migration chambers (24-well plates, 8- $\mu \mathrm{m}$ pore size. Corning Incorporated Costar, USA) at a density of $2 \times 10^{5}$ cells $/$ well, and incubated at $37^{\circ} \mathrm{C}$. A volume of $600 \mu \mathrm{l}$ of RPIM-1640 containing 10\% fetal bovine serum was added in the lower chamber. Wound healing assay was also carried out to detect the migration of cells and the two transfectant cells were cultured in 6-well plates until confluent. The cell layer was wounded using a sterile tip. After incubation for 24 to 36 hours, cells were photographed under a phase-contrast microscope. Experiments were carried out in triplicate. The distance between the two edges of the scratch (wound width) was measured at 10 sites in each image. The cell migration distance was determined by measuring the wound width at each time point from the wound width at the $0 \mathrm{~h}$ time point and then dividing by two [36].

For invasion assay, Matrigel matrix (BD, USA) was dissolved at $4^{\circ} \mathrm{C}$ overnight and then diluted with
RPIM- 1640. The upper chambers were coated with the diluted solution $(100 \mu \mathrm{l} /$ well $)$ and then placed at $37^{\circ} \mathrm{C}$ for $4 \mathrm{~h}$ to solidify the Matrigel matrix. The rest procedures were same with migration assay.

The migrating or invading cells in upper migration chambers were fixed with methanol for 15 minutes, and then stained with $0.1 \%$ crystal violet for 30 minutes. The unpenetrated cells on upper chamber were gently scraped from the surface with cotton swabs. Five visual fields $(200 \times$ magnification) were selected for each chamber by Image $\mathbf{J}$ software (Nikon, Japan) and the number of penetrated cells in these five fields $(200 \times$ magnification) was counted under an inverted microscope. Mean values of groups were recorded.

\section{Cell cycle analysis}

TPC-1 cells transfected with CCDC67 and control cells were harvested $72 \mathrm{~h}$ after culture, washed with cold phosphate buffered saline (PBS), and fixed in $1 \mathrm{ml}$ of $70 \%$ ethanol. After overnight incubation at $4^{\circ} \mathrm{C}$ in ethanol, cells were washed in PBS and suspended in $500 \mathrm{ml}$ propidine iodide (PI) 30 min before flow cytometry. Populations in G1, S, and G2 phases were measured by flow cytometry (Beckman, CA) and the data were analyzed using the Multicycle-DNA Cell Cycle Analyzed Software. The measurement was performed in triplicate.

\section{Detection of cell apoptosis}

TPC-1 cells transfected with CCDC67 and control cells were collected and fixed with $70 \%$ ethanol for the detection of apoptosis. The dyeing of cells was performed according to the instructions of Annexin V-FITC/PI cell apoptosis detection kit (SouthernBiotech, USA). Flow cytometry (BD Biosciences, USA) was used to detect the percentage of early apoptosis. The measurement was performed in triplicate.

\section{Statistical analysis}

The results are expressed as mean \pm the standard deviation (SD) of the mean of at least three separate experiments. Statistical significance was assessed using a two-tailed unpaired Student's $t$-test. The correlation between gene expression and potential causative variables were evaluated with the Chi-square test. $P<0.05$ was considered to indicate a statistically significant result. The statistical analyses were performed using the SPSS 17.0 software for Windows. GraphPad Prism 5 (GraphPad Software, USA) was used for graphs.

\section{ACKNOWLEDGMENTS AND FUNDING}

This study was supported by a grant from the National Natural Science Foundation of China (Grant 
Agreement No.81372863) and Innovation Scientists and Technicians Troop Construction Projects of Zhengzhou City (Grant Agreement No. 131PLJRC676).

\section{CONFLICTS OF INTEREST}

The authors declare that no competing financial interests exist.

\section{REFERENCES}

1. Xing M. Molecular pathogenesis and mechanisms of thyroid cancer. Nat Rev Cancer. 2013; 13:184-199.

2. Siegel R, Naishadham D, Jemal A. Cancer statistics for Hispanics/Latinos, 2012. CA Cancer J Clin. 2012; 62:283-298.

3. Yin DT, Wu W, Li M, Wang QE, Li H, Wang Y, Tang Y, Xing M. DKK3 is a potential tumor suppressor gene in papillary thyroid carcinoma. Endocr Relat Cancer. 2013; 20:507-514

4. Rahbari R, Kitano M, Zhang L, Bommareddi S, Kebebew E. RTN4IP1 is down-regulated in thyroid cancer and has tumor-suppressive function. J Clin Endocrinol Metab. 2013; 98:E446-E454.

5. Yin DT, Wang Q, Chen L, Liu MY, Han C, Yan Q, Shen R, He G, Duan W, Li JJ, Wani A, Gao JX. Germline stem cell gene PIWIL2 mediates DNA repair through relaxation of chromatin. PLoS One. 2011; 6:e27154.

6. Xing M. Genetic alterations in the phosphatidylinositol-3 kinase/Akt pathway in thyroid cancer. Thyroid. 2010; 20:697-706.

7. Paulikova S, Chmelarova M, Petera J, Palicka V, Paulik A. Hypermethylation of RAD51L3 and XRCC2 genes to predict late toxicity in chemoradiotherapy-treated cervical cancer patients. Folia Biol (Praha). 2013; 59:240-245.

8. Tamaki H, Sanda M, Katsumata O, Hara Y, Fukaya M, Sakagami H. Pilt is a coiled-coil domain-containing protein that localizes at the trans-Golgi complex and regulates its structure. Febs Lett. 2012; 586:3064-3070.

9. Burkhard P, Stetefeld J, Strelkov SV. Coiled coils: a highly versatile protein folding motif. Trends Cell Biol. 2001; 11:82-88.

10. Liu Z, Chen C, Yang H, Zhang Y, Long J, Long X, Fang W. Proteomic features of potential tumor suppressor NESG1 in nasopharyngeal carcinoma. Proteomics. 2012; 12:3416-3425.

11. Chen M, Ni J, Chang HC, Lin CY, Muyan M, Yeh S. CCDC62/ERAP75 functions as a coactivator to enhance estrogen receptor beta-mediated transactivation and target gene expression in prostate cancer cells. Carcinogenesis. 2009; 30:841-850.

12. Tsolakis AV, Grimelius L, Islam MS. Expression of the coiled coil domain containing protein 116 in the pancreatic islets and endocrine pancreatic tumors. Islets. 2012; 4:349-353.

13. Morra F, Luise C, Visconti R, Staibano S, Merolla F, Ilardi G, Guggino G, Paladino S, Sarnataro D, Franco R,
Monaco R, Zitomarino F, Pacelli R, et al. New therapeutic perspectives in CCDC6 deficient lung cancer cells. Int $\mathrm{J}$ Cancer. 2014.

14. Hanson D, Murray PG, O’Sullivan J, Urquhart J, Daly S, Bhaskar SS, Biesecker LG, Skae M, Smith C, Cole T, Kirk J, Chandler K, Kingston H, et al. Exome sequencing identifies CCDC8 mutations in 3-M syndrome, suggesting that CCDC8 contributes in a pathway with CUL7 and OBSL1 to control human growth. Am J Hum Genet. 2011; 89:148-153.

15. Novak DJ, Sabbaghian N, Maillet P, Chappuis PO, Foulkes WD, Tischkowitz M. Analysis of the genes coding for the BRCA1-interacting proteins, RAP80 and Abraxas (CCDC98), in high-risk, non-BRCA1/2, multiethnic breast cancer cases. Breast Cancer Res Treat. 2009; 117:453-459.

16. Zhong J, Zhao M, Luo Q, Ma Y, Liu J, Wang J, Yang M, Yuan X, Sang J, Huang C. CCDC134 is down-regulated in gastric cancer and its silencing promotes cell migration and invasion of GES-1 and AGS cells via the MAPK pathway. Mol Cell Biochem. 2013; 372:1-8.

17. Kobayashi S, Fukuhara A, Taguchi T, Matsuda M, Tochino Y, Otsuki M and Shimomura I. Identification of a new secretory factor, CCDC3/Favine, in adipocytes and endothelial cells. Biochem Biophys Res Commun. 2010; 392:29-35.

18. Park SJ, Jang HR, Kim M, Kim JH, Kwon OH, Park JL, Noh SM, Song KS, Kim SY, Kim YH, Kim YS. Epigenetic alteration of CCDC67 and its tumor suppressor function in gastric cancer. Carcinogenesis. 2012; 33:1494-1501.

19. Yin D, Xu J, Wang Y, Li H, Zhang Y, Jiang J, Wang Q. Expression of CCDC67 mRNA and its role in patients with papillary thyroid carcinoma [Article in Chinese]. Zhonghua Yi Xue Za Zhi. 2014; 94:2382-2385.

20. Kokona B, Rosenthal ZP, Fairman R. Role of the coiledcoil structural motif in polyglutamine aggregation. Biochemistry-Us. 2014; 53:6738-6746.

21. Lin RC, Scheller RH. Structural organization of the synaptic exocytosis core complex. Neuron. 1997; 19:1087-1094.

22. Meissner CS, Koppen-Rung P, Dittmer A, Lapp S, Bogner E. A "coiled-coil" motif is important for oligomerization and DNA binding properties of human cytomegalovirus protein UL77. PLoS One. 2011; 6:e25115.

23. Karunakaran V, Wickner W. Fusion proteins and select lipids cooperate as membrane receptors for the soluble N-ethylmaleimide-sensitive factor attachment protein receptor (SNARE) Vam7p. J Biol Chem. 2013; 288:28557-28566.

24. Nielsen BB, Kastrup JS, Rasmussen H, Holtet TL, Graversen JH, Etzerodt M, Thogersen HC, Larsen IK. Crystal structure of tetranectin, a trimeric plasminogenbinding protein with an alpha-helical coiled coil. Febs Lett. 1997; 412:388-396.

25. Zhu XJ, Liu J, Xu XY, Zhang CD, Dai DQ. Novel tumorsuppressor gene epidermal growth factor-containing fibulinlike extracellular matrix protein 1 is epigenetically silenced 
and associated with invasion and metastasis in human gastric cancer. Mol Med Rep. 2014; 9:2283-2292.

26. Baylin SB, Herman JG. DNA hypermethylation in tumorigenesis: epigenetics joins genetics. Trends Genet. 2000; 16:168-174.

27. Xing M. Recent advances in molecular biology of thyroid cancer and their clinical implications. Otolaryngol Clin North Am. 2008; 41:1135-1146.

28. Yin DT, Wang L, Sun J, Yin F, Yan Q, Shen R, He G, Gao JX. Association of the promoter methylation and protein expression of Fragile Histidine Triad (FHIT) gene with the progression of differentiated thyroid carcinoma. Int J Clin Exp Pathol. 2010; 3:482-491.

29. Ferraro A, Schepis F, Leone V, Federico A, Borbone E, Pallante P, Berlingieri MT, Chiappetta G, Monaco M, Palmieri D, Chiariotti L, Santoro M, Fusco A. Tumor suppressor role of the CL2/DRO1/CCDC80 gene in thyroid carcinogenesis. J Clin Endocrinol Metab. 2013; 98:2834-2843.

30. Bellelli R, Castellone MD, Garcia-Rostan G, Ugolini C, Nucera C, Sadow PM, Nappi TC, Salerno P, Cantisani MC, Basolo F, Gago TA, Salvatore G, Santoro M. FOXM1 is a molecular determinant of the mitogenic and invasive phenotype of anaplastic thyroid carcinoma. Endocr Relat Cancer. 2012; 19:695-710.
31. Nucera C. Targeting thyroid cancer microenvironment: basic research and clinical applications. Front Endocrinol (Lausanne). 2013; 4:167.

32. Antonello ZA, Nucera C. Orthotopic mouse models for the preclinical and translational study of targeted therapies against metastatic human thyroid carcinoma with BRAF(V600E) or wild-type BRAF. Oncogene. 2014; 33:5397-5404.

33. Liu D, Yang C, Bojdani E, Murugan AK, Xing M. Identification of RASAL1 as a major tumor suppressor gene in thyroid cancer. J Natl Cancer Inst. 2013; 105:1617-1627.

34. Zhang Y, Wang CP, Ding XX, Wang N, Ma F, Jiang JH, Wang QD, Chang JB. FNC, a novel nucleoside analogue, blocks invasion of aggressive non-Hodgkin lymphoma cell lines via inhibition of the Wnt/beta-catenin signaling pathway. Asian Pac J Cancer Prev. 2014; 15:6829-6835.

35. Xing M, Vasko V, Tallini G, Larin A, Wu G, Udelsman R, Ringel MD, Ladenson PW, Sidransky D. BRAF T1796A transversion mutation in various thyroid neoplasms. J Clin Endocrinol Metab. 2004; 89:1365-1368.

36. Feng Y, Sassi S, Shen JK, Yang X, Gao Y, Osaka E, Zhang J, Yang S, Yang C, Mankin HJ, Hornicek FJ, Duan Z. Targeting CDK11 in osteosarcoma cells using the CRISPRCas9 system. J Orthop Res. 2015; 33:199-207. 